\title{
Immunoactivative role of indoleamine 2,3-dioxygenase in gastric cancer cells in vitro
}

\author{
RUPENG ZHANG*, HUI LI*, JINPU YU, JINGZHU ZHAO, XUEJUN WANG, \\ GANG WANG, ZHOUJIA YAO, FENG WEI, QIANG XUE and XIUBAO REN \\ Tianjin Medical University Cancer Institute and Hospital, Tianjin 300060, P.R. China
}

Received June 11, 2010; Accepted November 19, 2010

DOI: $10.3892 / \mathrm{mmr} .2010 .398$

\begin{abstract}
To study the role of indoleamine 2,3-dioxygenase (IDO) in immune response and immune escape in gastric cancer, the human IDO gene was cloned by reverse transcriptionpolymerase chain reaction (RT-PCR) and inserted into the pIRES $_{2}$-EGFP vector to construct the IDO expression vector (pIRES ${ }_{2}$-EGFP-IDO). BGC-823 cells were transfected with the vector by electroporation and selected stable expression with G418. IDO expression was determined by RT-PCR and Western blot analysis. The enzymatic activity of IDO was estimated by determining tryptophan and kynurenine concentrations in the cell culture medium by an amino acid analyzer. To assess the effect of IDO on T cell-mediated cytotoxicity and proliferation, $\mathrm{T}$ cells from patients with gastric cancer were co-cultured with the IDO-transfected BGC-823 cells in the presence or absence of 1-MT, a competitive inhibitor of IDO. Cells transfected with the vector expressed high levels of IDO mRNA and protein, and a significantly higher level of kynurenic acid was detected in the culture medium of the transfected cells compared to the non-transfected cells $(\mathrm{P}<0.001)$. T cells co-cultured with the IDO-transfected cells exhibited significantly lower cytotoxicity compared to the control group $(\mathrm{P}<0.05)$. Additionally, IDO-transfected cells treated with 1-MT exhibited higher toxicity compared to the untreated IDO-transfected cells $(\mathrm{P}<0.01)$. We conclude that IDO plays a key role in gastric cancer immune suppression, possibly by inhibiting $\mathrm{T}$ cellmediated cytotoxicity and proliferation in vitro.
\end{abstract}

\section{Introduction}

Gastric cancer is a significant cause of mortality worldwide. Surgical resection, as the primary curative treatment, has

Correspondence to: Professor Xiubao Ren, Tianjin Medical University Cancer Institute and Hospital, Huanhuxi Road, Ti-Yuan-Bei, Hexi, Tianjin 300060, P.R. China

E-mail: rwziyi@yahoo.com

${ }^{*}$ Contributed equally

Key words: gastric cancer, tumor escape, indoleamine 2, 3-dioxygenase, $\mathrm{T}$ cell cytotoxicity, $\mathrm{T}$ cell proliferation limited efficacy to reduce mortality. However, immunotherapy for gastric cancer is still in its primary stages. Immune cells serve as key regulators in the microenvironment, which leads to both the prevention and enhancement of tumor growth. Recent studies have elucidated certain underlying mechanisms that contribute to tumor progression, including tumor antigenspecific immune tolerance, the tumor immunosuppressive microenvironment and tumor immune escape. However, the exact mechanisms are not fully understood.

Recently, considerable attention has been directed toward indoleamine 2,3-dioxygenase (IDO) due to its immunosuppressive function. IDO is the rate-limiting enzyme in the catabolism of tryptophan, an essential amino acid, and is widely distributed in various tissues of mammals. Certain studies have indicated that IDO may result in tryptophan depletion, and the depletion of tryptophan together with the accumulation of its metabolites may result in the suppression of $\mathrm{T}$ cell activation and proliferation $(1,2)$. The activity of IDO is partly reverted by the inhibitor, 1-methyltryptophan $(1-\mathrm{MT})$, in vivo $(3,4)$. However, the inhibitory effects of IDO have yet to be elucidated in gastric cancer.

To determine whether IDO plays a negative regulatory role in gastric cancer, we assessed IDO activity and the effect of 1-MT using a gastric cancer model in vitro.

\section{Materials and methods}

Cells and reagents. Peripheral blood mononuclear cells (PBMCs) were isolated from the peripheral blood of gastric cancer patients using the density gradient centrifugation method as previously described (5). The eukaryote expression vector pIRES $_{2}$-EGFP as well as the human breast cancer MDA-MB-435S and human gastric cancer BGC-823 cell lines were obtained from the Department of Immunology, Tianjin Medical University Cancer Institute and Hospital, Tianjin, China. Primers were synthesized by Takara (Japan), and mouse anti-human IDO monoclonal antibody was purchased from Chemicon (USA). Mouse anti-human $\beta$-actin monoclonal antibody was purchased from Santa Cruz Biotechnology (Santa Cruz, CA, USA), and L-tryptophan, L-kynurenine and 1-MT were purchased from Sigma (St. Louis, MO, USA). The Olerup SSP HLA-A kit was purchased from Qiagen (Germany).

IDO gene cloning. Total RNA was isolated from human breast cancer MDA-MB-435S cells, which express high levels of 
IDO, using TRIzol reagent (Invitrogen, Carlsbad, CA, USA) according to the manufacturer's instructions. Complementary cDNA was synthesized using the high fidelity PrimeScript ${ }^{\mathrm{TM}}$ reverse transcription-polymerase chain reaction (RT-PCR) kit (Takara, Japan). The primer design was based on the protein coding region of the IDO gene: forward primer, 5'-AGATCTGCCACCATGGCACACGCTATGGAAAAC-3' and reverse primer, 5'-GTCGACTTAACCTTCCTTCAAAAG GGATTTC-3'. Amplification products were purified with a PCR product purification kit (Takara). The pMD19-T vector and PCR product were ligated using the Takara DNA Ligation kit (Takara), and the recombinant plasmid was transformed into competent Escherichia coli DH5 $\alpha$ cells by electroblotting according to the manufacturer's instructions. Plasmid DNA of a single clone was isolated with the EndoFree Plasmid kit (Tianfen Biotech, Beijing, China). The plasmid was sequenced to verify the accuracy of the restriction analysis results, and the recombinant vector was named IDO/T.

Construction of the expression vector pIRES $S_{2}-E G F P$-IDO. The plasmids IDO/T and pIRES $_{2}$-EGFP were isolated and digested with $B g I I I$ and SalI restriction enzymes, and the resulting DNA fragments of the target gene and pIRES $_{2}$-EGFP were purified and ligated with T4 DNA ligase (mole ratio 3:1; New England Biolabs, USA). The recombinant vector ( pIRES $_{2}$-EGFP-IDO) was transformed into competent $E$. coli $\mathrm{DH} 5 \alpha$ cells by electroblotting, and its accuracy was verified by subcloning and sequencing. Plasmid DNA was isolated using the Qiagen EndoFree plasmid mini kit (Qiagen, Beijing, China), and the DNA quantity was determined by the DU 640 nucleic acid protein analyzer (Beckman Coulter, USA). Optical density $\mathrm{OD}_{260} / \mathrm{OD}_{280}$ readings were in the range of 1.8-2.0.

Cell culture and transfection. Human gastric cancer BGC-823 cells were cultured in RPMI-1640 medium (Gibco, Invitrogen) containing 10\% fetal bovine serum (FBS; Hyclone, Logan, UT, USA) at $37^{\circ} \mathrm{C}$ with $5 \% \mathrm{CO}_{2}$. Prior to transfection, the cells were digested with trypsin, centrifuged, and resuspended in $0.4 \mathrm{ml}$ electroporation buffer (Eppendorf AG, Hamburg, Germany) at $1 \times 10^{6} / \mathrm{ml}$. pIRES ${ }_{2}$-EGFP-IDO $(4-8 \mu \mathrm{g})$ was combined with the cells, and electroporation was performed with single pulses of $560 \mathrm{~V}$ for $60 \times 10^{-6} \mathrm{sec}$. The pIRES $_{2}$-EGFP empty vector was used as a transfection control. Following electroporation, the cells were transferred into 6-well culture plates, and the transfection efficiency was determined by G418 screening. To obtain stable clones, $400 \mu \mathrm{g} / \mathrm{ml} \mathrm{G} 418$ was used as the initial concentration for screening in the first week and $200 \mu \mathrm{g} / \mathrm{ml}$ for the next 2 weeks.

Determining IDO mRNA expression by RT-PCR. RNA was extracted from the cultured cells using TRIzol reagent according to the manufacturer's instructions. Following isolation, Moloney murine leukemia virus reverse transcriptase was used in the cDNA synthesis with the total RNA templates. The $20-\mu 1$ PCR reaction contained $5 \mu 1$ of $10 \mathrm{X}$ buffer, $0.5 \mu \mathrm{l}$ cDNA, $1 \mu \mathrm{l}$ each of the forward and reverse primers, $1.2 \mu \mathrm{l}$ of $25 \mathrm{mM} \mathrm{MgCl}, 2.5 \mathrm{mM}$ dNTPs and $0.5 \mu \mathrm{l} \mathrm{Taq}$ polymerase. The IDO primers were designed to amplify a 188-bp fragment: forward primer, 5'-CATCTGCAAATCGTGACTAAG-3' and reverse primer, 5'-CAGTCGACACATTAACCTTCCTTC-3'. $\beta$-actin was used as an internal control: forward primer, 5'-CATCTGCAAATCGTGACTAAG-3' and reverse primer, 5'-CAGTCGACACATTAACCTTCCTTC-3'. The amplification procedure was as follows: denaturing at $94^{\circ} \mathrm{C}$ for $3 \mathrm{~min}$ and 30 cycles each at $94^{\circ} \mathrm{C}$ for $60 \mathrm{sec}$, annealing at $58^{\circ} \mathrm{C}$ for $30 \mathrm{sec}$ and extension at $72^{\circ} \mathrm{C}$ for $60 \mathrm{sec}$, followed by a final extension at $72^{\circ} \mathrm{C}$ for $10 \mathrm{~min}$. The amplification products were identified by $1.0 \%$ agarose gel electrophoresis, and the images were captured and analyzed by the Bio-Rad gel imaging system (Bio-Rad, USA). IDO mRNA expression was normalized to $\beta$-actin mRNA.

Western blot analysis. Cell lysates were obtained by adding cell lysis solution (Pierce, USA) to the cultured cells. The proteins were separated by $10 \%$ sodium dodecyl sulfatepolyacrylamide gel electrophoresis (SDS-PAGE) and electrophoretically transferred to polyvinylidene fluoride membranes. The membranes were blocked overnight in 5\% fat-free milk in Tris-buffered saline with Tween and then incubated with affinity purified mouse anti-human IDO IgG (1:500 dilution) overnight at $4^{\circ} \mathrm{C}$. After being washed in phosphate-buffered saline, the membrane was incubated with horseradish peroxidase-linked goat anti-mouse $\operatorname{IgG}(1: 5,000)$ (Zhongshanjinqiao, Beijing, China) for $1 \mathrm{~h}$ at room temperature. The immunopositive bands were visualized using SuperSignal West Pico Chemiluminescent Substrate (Pierce).

IDO determined by amino acid analyzer. The cells transfected with the IDO expression vector and empty vector controls were incubated in RPMI-1640 medium containing $10 \% \mathrm{FBS}$ at $37^{\circ} \mathrm{C}$ with $5 \% \mathrm{CO}_{2}$. The culture medium was collected after a $72-\mathrm{h}$ incubation, and $2 \mathrm{ml}$ of medium was analyzed using the Hitachi L-8800 automatic amino acid analyzer (Hitachi, Japan).

Determination of the inhibition of $T$ lymphocyte proliferation. PBMCs from gastric cancer patients were separated and collected using density gradient centrifugation method. The cells were incubated with RosetteSep Human CD3 Depletion Cocktail (RosetteSep, USA), and $\mathrm{CD}^{+}{ }^{+} \mathrm{T}$ cells were collected and used. Four groups of BGC-823 cells were used in this experiment: i) pIRES $_{2}$-EGFP-IDO-transfected cells, ii) pIRES $_{2}$-EGFP-IDO-transfected cells treated with the IDO competitive inhibitor 1-MT (final concentration $1 \mathrm{mM}$ ), iii) pIRES $_{2}$-EGFP-transfected cells (empty vector transfection) and iv) non-transfected BGC-823 cells. The experimental groups were stimulated with $30 \mathrm{~Gy} \mathrm{X}$-ray irradiation. The concentration gradients of the cell groups were $1 \times 10^{6}, 5 \times 10^{5}$, $2.5 \times 10^{5}$ and $1.25 \times 10^{5}$ cells $/ \mathrm{ml}$, respectively. The cells were then combined with $1 \times 10^{6}$ cells $/ \mathrm{ml} \mathrm{CD} 3^{+} \mathrm{T}$ cells in $96-$ well plates $(200 \mu \mathrm{l} /$ well). After a $72-\mathrm{h}$ incubation, the cells were treated with 3-(4,5-dimethylthiazol-2-yl)-2,5-diphenyltetrazolium bromide (MTT; Sigma-Aldrich), and the absorbance (A) was measured by a microplate reader at $492 \mathrm{~nm}$. T cell suppression was calculated as: rate $=\left(1-\mathrm{A}_{\text {Experimental group }} / \mathrm{A}_{\text {Control }}\right.$ group). The experiments were performed in triplicate (three wells/treatment group).

Cytotoxicity of $T$ cells co-cultured with gastric cancer BGC-823 cells. HLA-A24+ PBMCs from gastric cancer patients were selected using an HLA-A kit (Qiagen). T cells 
A

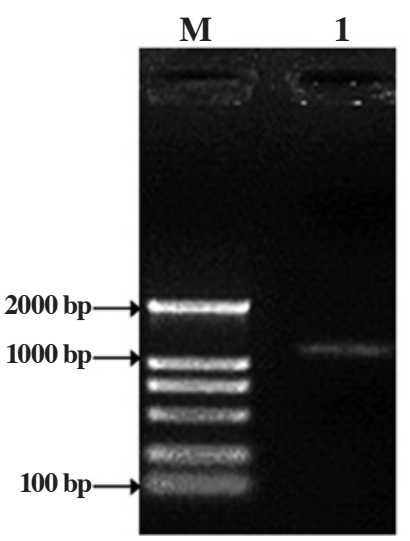

B

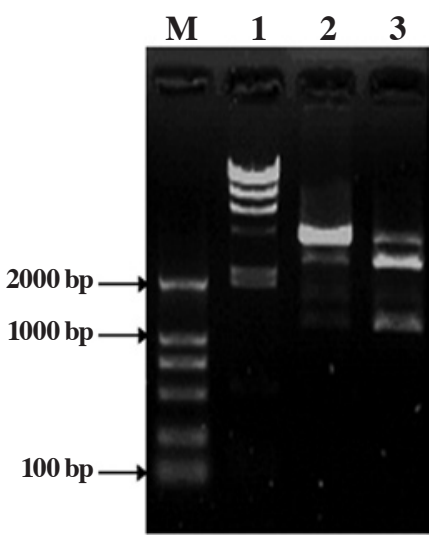

Figure 1. Analysis of IDO expression of the IDO/T-transfected cells. PCR amplification products were separated by $1.0 \%$ agarose gel electrophoresis. (A) Lanes: M, DNA marker DL2000; 1, polymerase chain reaction product. (B) Restriction analysis of the IDO/T vector. Lanes: 1, DNA marker DL2000; 2, $\lambda$-HindIII DNA marker; 3, IDO/T; 4, BglII/SalI restriction digestion.

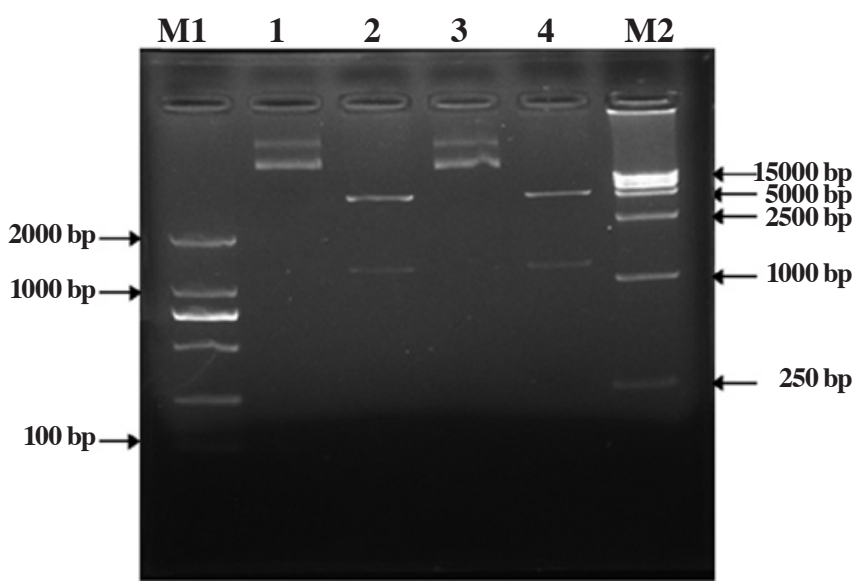

Figure 2. Analysis of the pIRES $_{2}$-EGFP-IDO expression vector. Lanes: M1, DNA marker DL2000; M2, DNA marker DL15000; 1 and 3, pIRES $_{2-}$ EGFP-IDO expression vector; 2 and 4 , pIRES $_{2}$-EGFP-IDO digested by BglII/SalI.

were added in order to achieve a ratio of effector $\mathrm{T}$ cells to target cells (HLA-A24+ PBMCs) of 40:1, 20:1 and 10:1 (the final concentration of the effector $\mathrm{T}$ cells and the target cells were $1 \times 10^{6}$ and $1 \times 10^{5}$ cells $/ \mathrm{ml}$, respectively). BGC-823 cells, divided into the same experimental groups as described, were co-cultured with the effector and target cells in a 96-well plate (three replicate wells/treatment group). The cytotoxicity of the effector T cells was determined using a lactate dehydrogenase kit (Promega, Madison, WI, USA), and A at $492 \mathrm{~nm}$ was determined by using a microplate reader. The cytotoxic activity of the $\mathrm{T}$ lymphocytes was determined as follows: $\left(\mathrm{A}_{\text {Experimental group }}\right.$ - $\left.\mathrm{A}_{\text {Spontaneous release group }}\right) /\left(\mathrm{A}_{\text {Maximum release group }}-\mathrm{A}_{\text {Spontaneous release group }}\right)$.

Statistical analysis. Statistical analysis was conducted using SPSS v13.0 software (SPSS Inc., Chicago, IL, USA). Data were analyzed using the Student's t-test or one-way analysis of variance followed by Student-Newman-Keuls multiple comparisons procedure. $\mathrm{P}<0.05$ was defined as statistically significant.

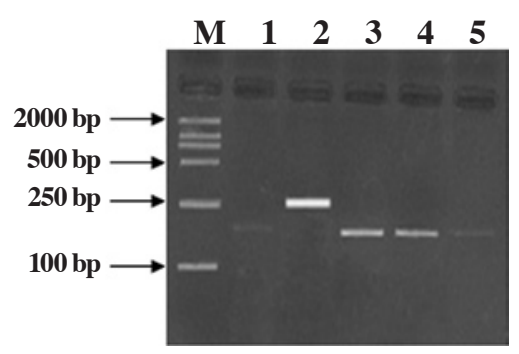

Figure 3. Reverse transcription-PCR analysis of exogenous IDO expression. Lanes: M, DNA marker DL2000; 1, non-transfected BGC-823 cells; $2, \beta$-actin internal control; 3 and 4 , IRES $_{2}$-EGFP-IDO-transfected cells; 5 , pIRES $_{2}$-EGFP-transfected cells (empty vector).

\section{Results}

Recombinant vectors were verified by restriction enzyme digestion and sequencing. The IDO gene was successfully cloned by RT-PCR (Fig. 1A). The IDO/T and pIRES ${ }_{2}$-EGFP-IDO constructs were analyzed by restriction digestion with $B g l \mathrm{II}$ and SalI, and the accuracy of the clones was then verified by sequencing. The results confirmed that the IDO/T vector contained the expected 1,225-bp protein coding sequence and

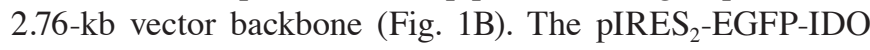
recombinant vector contained the 1,225 -bp protein coding sequence and the 5.4-kb vector backbone (Fig. 2).

Electroporation-mediated gene transfer. Human gastric cancer BGC-823 cells transfected with pIRES $_{2}$-EGFP demonstrated strong and uniformly distributed green fluorescence at $24 \mathrm{~h}$, indicating successful electroporation-mediated gene transfer. However, $48 \mathrm{~h}$ after pIRES $_{2}$-EGFP-IDO transfection, the cells exhibited increased cytoplasmic granules and became round and enlarged. Vacuoles in the cytoplasm and massive cell death were observed in this group of cells, although some displayed uniformly distributed green fluorescence (data not shown).

IDO mRNA expression. IDO mRNA expression in BGC-823 cells was determined by RT-PCR. Levels of IDO mRNA were significantly increased in the pIRES $_{2}$-EGFP-IDO-transfected BGC-823 cells (one-way ANOVA; $\mathrm{F}=29.638, \mathrm{P}<0.001$ ) (Fig. 3). Mean optical density of IDO/ $\beta$-actin in the non-transfected, pIRES $_{2}$-EGFP-transfected and pIRES $_{2}$-EGFP-IDO-transfected cells were $0.1190 \pm 0.0547,0.1183 \pm 0.0602$ and $0.5627 \pm 0.1414$, respectively.

Exogenous IDO protein expression. Western blot analysis was used to determine IDO protein expression in the BGC-823 cells transfected with pIRES $_{2}$-EGFP-IDO. Protein was observed in the transfected cells, indicating expression of exogenous IDO. This protein was not observed in the control or empty vector-transfected cell groups (Fig. 4).

IDO activity in the transfected cells. To determine the IDO activity in the transfected cells, tryptophan concentrations in the culture medium were measured using an amino acid automatic analyzer. The tryptophan concentrations were $3.23 \pm 0.53$ and $6.03 \pm 0.51 \mathrm{mg} / \mathrm{l}$ in the pIRES $_{2}$-EGFP-IDO-transfected 


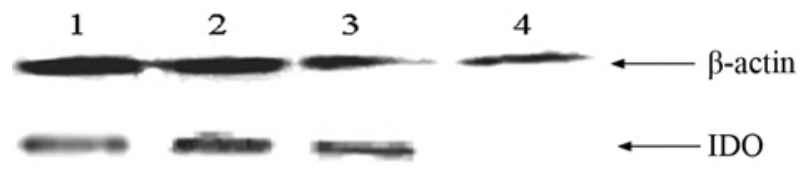

Figure 4. Western blot analysis of exogenous IDO protein expression. Upper panel, $\beta$-actin (45 kDa); lower panel, IDO (42 kDa). Lanes 1-3, pIRES $_{2}$-EGFP-IDO-transfected cells; 4, non-transfected cells.

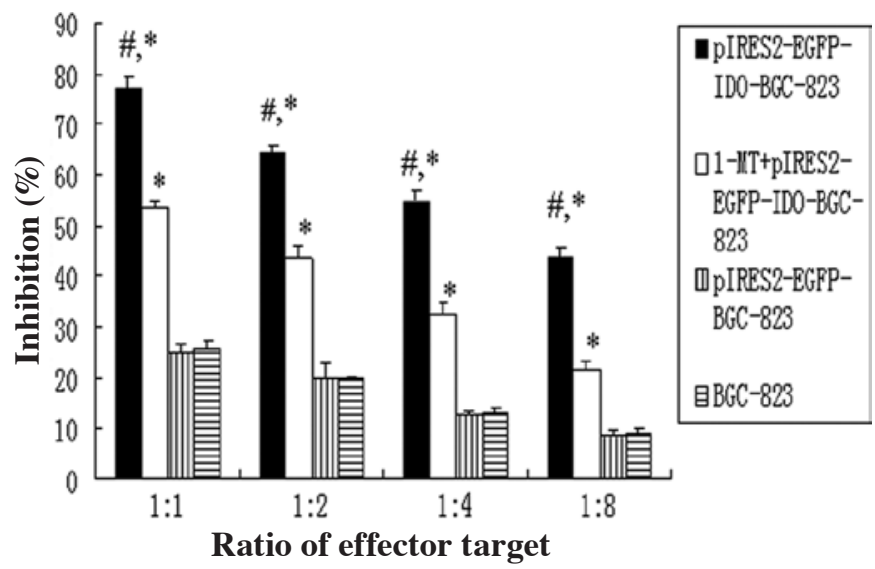

Figure 5. Suppression of T lymphocyte proliferation by IDO-overexpressing BGC- 823 cells. Bars represent the mean \pm SD. ${ }^{*} \mathrm{p}<0.05$, compared to $\mathrm{pIRES}_{2}-$ EGFP-IDO-transfected BGC-823 cells with 1-MT treatment; "p $<0.05$, compared to pIRES $_{2}$-EGFP-transfected or untransfected BGC-823 cells.

and non-transfected cell groups, respectively $(\mathrm{t}=13.32$, $\mathrm{P}<0.001)$. A significant increase in kynurenine concentration in the pIRES 2 -EGFP-IDO-transfected cell group was also observed, compared to the non-transfected group. Kynurenine concentrations were $4.84 \pm 0.11$ and $1.83 \pm 0.10 \mathrm{mg} / 1$ in the pIRES $_{2}$-EGFP-IDO-transfected and non-transfected cell groups, respectively $(\mathrm{t}=42.916, \mathrm{P}<0.001)$.

Effect of IDO on the proliferation of $T$ lymphocytes. To determine the inhibitory effect of IDO on T lymphocyte proliferation, the following experimental groups were used: BGC-823 cells transfected with pIRES $_{2}$-EGFP-IDO (with or without 1-MT treatment), BGC-823 cells transfected with pIRES $_{2}$-EGFP (empty vector) and non-transfected cells. The data showed statistically differential inhibitory effects among the experimental groups ( $\mathrm{F}=13.720, \mathrm{P}<0.001)$ (Fig. 5). Inhibition of $\mathrm{T}$ lymphocytes was greater in the pIRES $_{2}$-EGFP-IDO-transfected cells with or without 1-MT treatment compared with the pIRES 2 -EGFP-transfected and non-transfected cell groups. Furthermore, the untreated pIRES $_{2}$-EGFP-IDO-transfected cells demonstrated greater inhibition compared to the 1-MTtreated cells $(\mathrm{q}=3.926, \mathrm{P}<0.05)$.

Effect of IDO on the cytotoxic activity of T lymphocytes. The cytotoxic activity of the $\mathrm{T}$ cells cultured with gastric cancer BGC-823 cells against human PBMCs was assessed. Significantly lower cytotoxic $\mathrm{T}$ lymphocyte activity was observed in the pIRES $_{2}$-EGFP-IDO-transfected cells with or without 1-MT, compared to the pIRES2-EGFP-transfected and non-transfected cell groups $(\mathrm{F}=12.122, \mathrm{P}<0.01)$ (Fig. 6). Furthermore, the 1-MT-treated IRES $_{2}$-EGFP-IDO-transfected

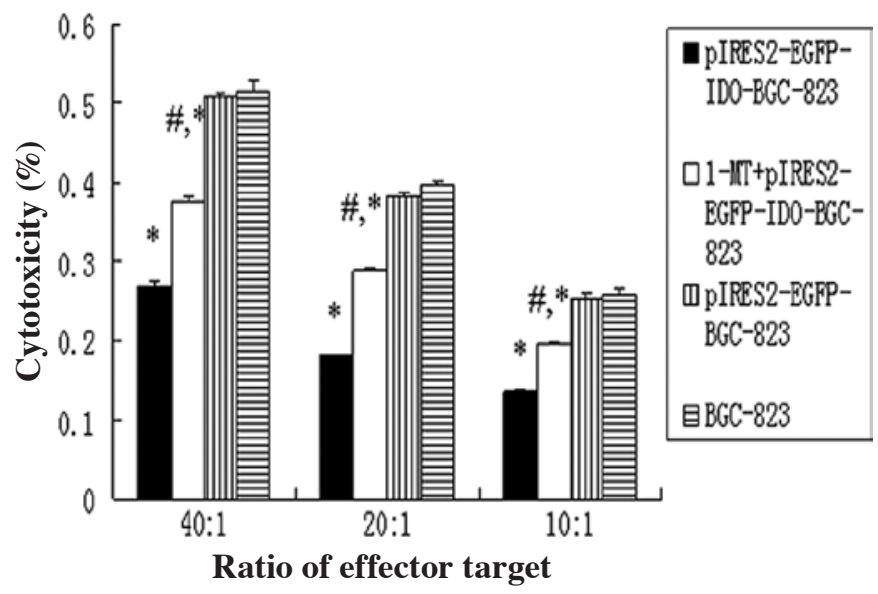

Figure 6. Cytotoxic activity of T cells co-cultured with IDO-overexpressing gastric cancer BGC-823 cells. Bars represent the mean $\pm \mathrm{SD}$. ${ }^{*} \mathrm{p}<0.05$, compared to pIRES $_{2}$-EGFP-IDO-transfected BGC-823 cells without 1-MT treatment; ${ }^{*} \mathrm{p}<0.05$, compared to pIRES $_{2}$-EGFP-transfected or untransfected BGC- 823 cells.

cells demonstrated significantly higher cytotoxic $\mathrm{T}$ lymphocyte activity than the untreated cells ( $\mathrm{q}=3.888, \mathrm{P}<0.05)$.

\section{Discussion}

IDO is a critical enzyme in L-tryptophan catabolism through the kynurenine pathway. Previous studies have demonstrated that IDO plays a key role in the abnormal cellular immune function of $\mathrm{T}$ lymphocytes in cancer patients (6). It also contributes to tumor-induced tolerance by direct suppression of $\mathrm{T}$ cells. However, the mechanism underlying these effects remains unclear. IDO is thought to initiate the degradation of tryptophan in the kynurenine pathway, which produces metabolites that modulate $\mathrm{T}$ cell toxicity. In addition, IDO depletes tryptophan in the tumor microenvironment, thereby inhibiting $\mathrm{T}$ cell proliferation $(7,8)$. The combined effects of tryptophan starvation and tryptophan catabolites appear to be crucial in the induction of immune tolerance. Furthermore, IDO secreted by tumor cells may induce regulatory $\mathrm{T}$ cells in the tumor microenvironment. These may be specific events that contribute to the induction of tumor immune escape by IDO.

Tumor cells transfected with IDO overexpress the protein. This provides a useful tool for assessing the influence of IDO on the immune responses of cancer cells. Studies have revealed that IDO expression is induced in the tumor cells of invasive carcinoma, tumor-adjacent tissue, lymph nodes and in antigen-presenting cells (including dendritic cells). IDO induces an immunosuppressive effect against $\mathrm{T}$ cells, indirectly suppressing $\mathrm{T}$ cell-mediated cytotoxicity (9).

Uyttenhove et al (4) found that the majority of tumor cells, including lung, prostate, pancreatic and cervical cancers, express IDO, thereby suppressing the activity of local $\mathrm{T}$ cells and protecting tumor tissues from the immune system. In this study, we cloned the IDO gene and constructed a eukaryotic expression vector. We hypothesized that cells transfected with this vector would suppress the proliferation of $\mathrm{T}$ cells by depleting tryptophan in the local microenvironment. This hypothesis was tested in human gastric cancer BGC-823 cells, and the culture medium of the transfected BGC-823 cells was 
found to have significantly decreased tryptophan and higher levels of kynurenic acid, indicating that the exogenously expressed IDO possessed enzymatic activity and catalyzed tryptophan metabolism in vitro. These preliminary findings allow for further investigation of the interaction between IDO and tumor cells in regards to immune response.

In the present study, gastric cancer BGC-823 cells were transfected with IDO and treated with the IDO inhibitor 1-MT in order to determine the effects of IDO on T cell activity and proliferation. We observed lower $\mathrm{T}$ cell proliferation and cytotoxicity in IDO-overexpressing cells with or without 1-MT treatment compared to the control groups. However, 1-MT treatment of IDO-transfected cells restored some of the T cell proliferation and cytotoxicity, confirming that the immunosuppressive effects were exerted by IDO.

Previous studies have reported improved therapeutic effects using the combination of 1-MT and chemotherapy compared to chemotherapy alone (10). However, it was reported that the inhibition of T cell proliferation and activity was not completely reversed by 1-MT, similar to the results of the present study, in which 1-MT attenuated, but did not completely block, IDO activity. Expression of IDO in tumor cells may not be the sole means of immune escape in host cells. Antigen-presenting cells also appear to contribute to tumor immunosuppression (8). Therefore, the action and optimal concentration of 1-MT in IDO inhibition requires further investigation. Further studies on the mechanisms of IDO-induced tumor immunosuppression and regulation of IDO activity by 1-MT may provide useful information for gastric cancer immunotherapy.

In conclusion, the present study demonstrated that IDO plays a key role in gastric cancer immune suppression, possibly by inhibiting $\mathrm{T}$ cell-mediated cytotoxicity and proliferation in vitro, and 1-MT may partly reverse the activity of IDO. These results provide insight into the function of IDO in the induction of immune escape, and indicate that 1-MT has the potential to serve as a novel therapeutic candidate for gastric cancer.

\section{Acknowledgements}

This study was supported by grants from the Tianjin Health Bureau Funds of China (no. 05-KYZ86).

\section{References}

1. Munn DH and Mellor AL: Indoleamine 2,3-dioxygenase and tumor-induced tolerance. J Clin Invest 117: 1147-1154, 2007.

2. Fallarino F, Grohmann U, You S, et al: The combined effects of tryptophan starvation and tryptophan catabolites down-regulate $\mathrm{T}$ cell receptor zeta-chain and induce a regulatory phenotype in naive T cells. J Immunol 176: 6752-6761, 2006.

3. Hou W, Li S, Wu Y, Du X and Yuan F: Inhibition of indoleamine 2,3-dioxygenase-mediated tryptophan catabolism accelerates crescentic glomerulonephritis. Clin Exp Immunol 156: 363-372, 2009.

4. Uyttenhove C, Pilotte L, Théate I, et al: Evidence for a tumoral immune resistance mechanism based on tryptophan degradation by indoleamine 2,3-dioxygenase. Nat Med 9: 1253-1254, 2003.

5. Yu J, Ren X, Cao S, Zhang W and Hao X: Th1 polarization and apoptosis-inducing activity of $\mathrm{CD}^{+}{ }^{+} \mathrm{T}$-cells in cytokine-induced killers might favor the antitumor cytotoxicity of cytokineinduced killers in vivo. Cancer Biother Radiopharm 21: 276-284, 2006.

6. Gajewski TF, Meng Y and Harlin H: Immune suppression in the tumor microenvironment. J Immunother 29: 233-240, 2006.

7. Fallarino F, Grohmann U, Vacca C, et al: T cell apoptosis by tryptophan catabolism. Cell Death Differ 9: 1069-1077, 2002.

8. Belladonna ML, Grohmann U, Guidetti P, et al: Kynurenine pathway enzymes in dendritic cells initiate tolerogenesis in the absence of functional IDO. J Immunol 177: 130-137, 2006.

9. Forouhar F, Anderson JL, Mowat CG, et al: Molecular insights into substrate recognition and catalysis by tryptophan 2,3dioxygenase. Proc Natl Acad Sci USA 104: 473-478, 2007.

10. Hou DY, Muller AJ, Sharma MD, et al: Inhibition of indoleamine 2,3-dioxygenase in dendritic cells by stereoisomers of 1-methyltryptophan correlates with antitumor responses. Cancer Res 67: 792-801, 2007 